\title{
Projeto Cata-Sopro: Um Joystick de Sopro Baseado em Sensores Eletromagnéticos
}

\author{
Igor Garcia $^{1}$, Rafael Araújo ${ }^{1}$, Victor T. Sarinho ${ }^{1}$ \\ ${ }^{1}$ Laboratório de Entretenimento Digital Aplicado - LEnDA \\ Universidade Estadual de Feira de Santana - UEFS \\ Feira de Santana - Bahia - Brasil \\ \{igonildo7,rafaelaraujo.gcc\}@gmail.com, vsarinho@uefs.br
}

\begin{abstract}
Nowadays, there is a demand of encouraging the blowing exercise in a playful way. This paper presents the development process of Cata-Sopro, a videogame control prototype that has the blowing exercise its main function. For this, the hardware scheme, operational logic control and preliminary tests results are described. As a result, despite the current demands for extra functional tests and aesthetic improvements, a simple and low cost apparatus was provided, being able to be applied in speech therapies aimed to stimulate desired orofacial exercises.
\end{abstract}

Resumo. Atualmente, existe a necessidade de incentivar o exercício de sopro de maneira lúdica. Este artigo apresenta o processo de desenvolvimento do CataSopro, um protótipo de controle de videogame que possui como função principal o exercício de sopro. Para tal, esquema de hardware utilizado, controle lógico de funcionamento e os resultados de testes preliminares são descritos. Como resultado, apesar das demandas por testes adicionais de funcionamento e melhorias estéticas, um aparelho simples e de baixo custo foi produzido, capaz ser aplicado em terapias fonoaudiologias voltadas para a estimulação de exercícios orofaciais desejados.

\section{Introdução}

Terapias fonoaudiológicas buscam promover o tratamento das alterações musculares e funcionais orofaciais de pacientes em geral [Comin and Passos Filho 1999], através de exercícios realizados durante as sessões de terapia que costumam ser repetitivos, cansativos e por várias vezes monótonos.

Jogos digitais têm se tornado uma forte tendência em nível mundial para fins relacionados a saúde, abrindo espaço para o uso de jogos sérios em novas abordagens de tratamento de síndromes e doenças existentes [Clua 2014]. Eles também oferecem um meio promissor para o usuário interagir de forma natural e intuitiva com ambientes computacionais, através da aquisição e do processamento de sinais fisiológicos diversos, explorando assim novas capacidades de comunicação homem-computador passíveis de uso [Arroyo-Palacios and Romano 2009].

Este artigo apresenta o Cata-Sopro, um joystick que permite a execução de comandos de controle de jogos digitais tendo como base a variação de intensidade em sensores eletromagnéticos gerada pelo ato de soprar. Dessa forma, por ter caráter exploratório, essa pesquisa tem como objetivo proporcionar, através da aquisição e processamento de sinais 
fisiológicos, uma jogabilidade diferenciada voltada para jogos sérios de saúde, neste caso específico para terapias fonoaudiológicas baseadas no sopro.

\section{Trabalhos Relacionados}

Algumas abordagens têm sido aplicadas na monitoração de sinais relacionados ao sopro humano. Como exemplo, BLUI [Patel and Abowd 2007] fornece uma interface que interpreta o ato de soprar em um laptop ou tela de computador para controlar diretamente algumas aplicações interativas (Figura 1(a)). Uma Interface Respiratória-Computador (RCI), a qual foi avaliada por um mini-jogo onde os jogadores participavam de uma corrida para explodir balões virtuais em 3D [Arroyo-Palacios and Romano 2009], também foi desenvolvida (Figura 1(b)). Blowatch [Chen 2015] fornece um método de entrada para smartwatches que utiliza sopros e gestos para invocar varias operações do dispositivo, tais como ajustar o volume da música, tirar uma foto ou atender uma chamada telefônica (Figura 1(c)). Cornestick [Sarinho 2017] apresenta o desenvolvimento de um joystick de sopro para jogos digitais que captura movimentos direcionais e de sopro em uma corneta através de sensores Arduino (Figura 1(d)). Para finalizar, Umistick [Alves et al. 2018] apresenta o desenvolvimento de um joystick de sopro para jogos digitais que captura movimentos direcionais e de sopro através de um sensor Arduíno de temperatura e umidade (Figura 1(e)).

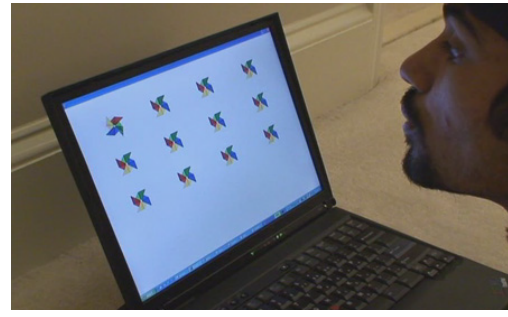

(a) BLUI

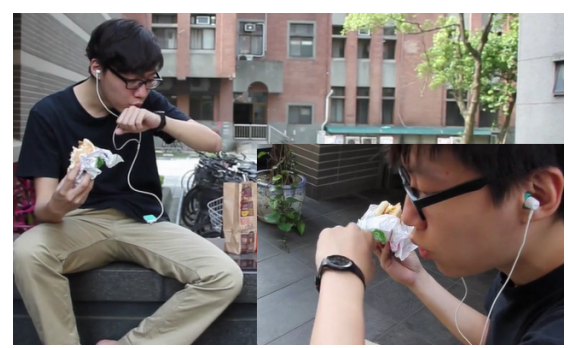

(c) Blowatch

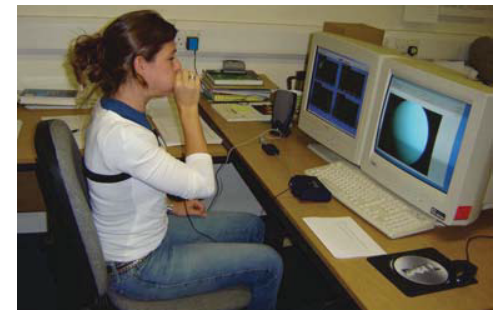

(b) RCI

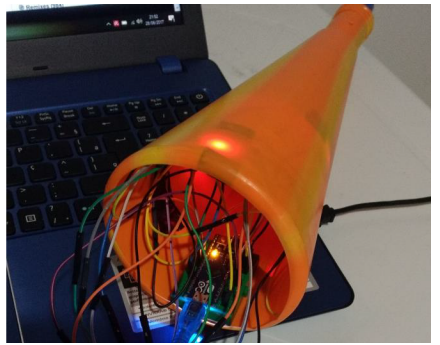

(d) Cornestick

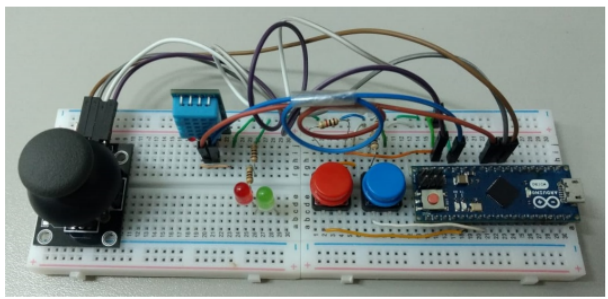

(e) Umistick

Figura 1. Tecnologias desenvolvidas com base na monitoração do sopro. 


\section{Metodologia}

Para o projeto Cata-Sopro, pretende-se aplicar o uso de ímãs como foco do mecanismo de captação dos dados de sopro. A ideia é produzir um cata-vento com uma ventoinha movida pelo sopro e uma haste oca de plástico para suporte do circuito interno do joystick, neste caso o direcional analógico na parte frontal controlado pelo polegar e um botão na traseira pressionável pelo o dedo indicador. Um sensor eletromagnético, acoplado próximo às hélices de um cooler (ventoinha) onde se encontram os ímãs, terá a função de captar as variações do campo eletromagnético proporcionadas pelo ato de soprar e transferir para o jogo. Desta forma, para compor a parte física do respectivo joystick, foram utilizados:

- 1 Arduíno micro, responsável pelo controle geral do joystick proposto (Figura 2(a));

- 1 alavanca analógica de três eixos, responsável pela captura dos movimentos direcionais do jogador, retornando valores referentes às teclas do teclado (W, A, S, D) para representar os movimentos direcionais em um jogo (cima, esquerda, baixo, direita) (Figura 2(c));

- 1 sensor eletromagnético de 3 eixos modelo MAG 3110, utilizado para captar variações magnéticas induzidas pelo ato de soprar (Figura 2(b));

- 1 cooler com 1 suporte para anexo de 2 imãs, capaz de gerar um campo magnético variável a partir do sopro do jogador (Figura 2(b));

- 1 botão de pressão (push-button) comum, para habilitar em conjunto com o sensor de sopro a ativação de eventos especiais (tiro, salto, etc.) (Figura 2(a)); e

- 1 resistor $(10 \mathrm{k} \mathrm{ohm})$, para complementar a configuração do circuito elétrico prototipado (Figura 2(a)).

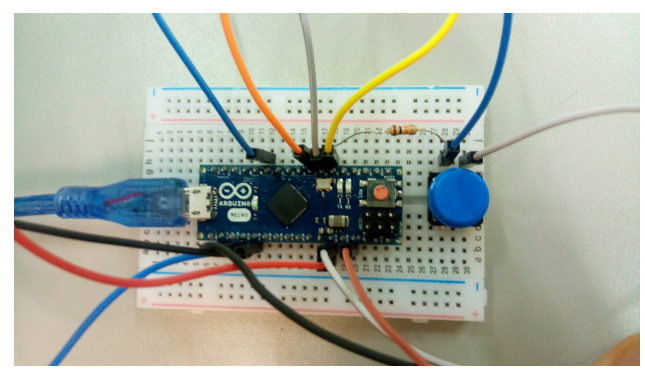

(a) Protótipo

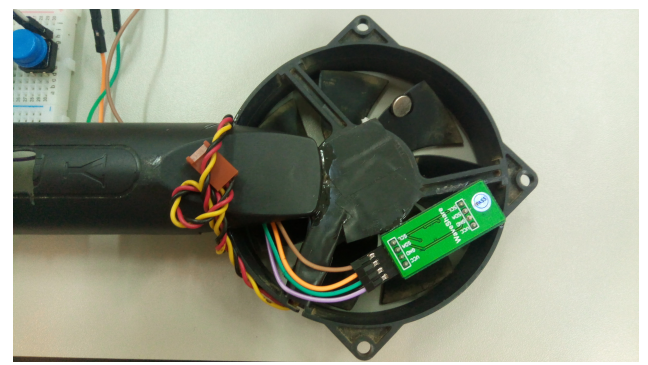

(b) Ventoinha

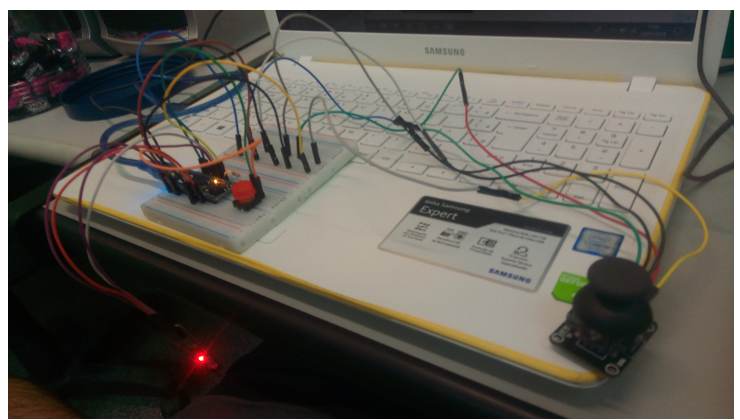

(c) Direcional

Figura 2. Elementos de hardware integrados em protoboard para o Cata-Sopro. 
No que tange ao código de controle aplicado no Arduíno, este realiza a ativação dos sensores com a execução de dois procedimentos base, o setup e o loop (Figura 3). No setup são inicializadas algumas variáveis, bem como a emulação do teclado, limpeza do buffer e definição de pinos de entrada e saída. No procedimento loop, é efetuada: a leitura constante dos valores recebidos pelos pinos de entrada; a execução do código de controle do joystick; e o envio das saídas de indicação de comandos do joystick para o jogo, neste caso as teclas do teclado a serem emitidas ou um LED que deve ser ativado em determinados momentos.

Com relação a lógica de controle do joystick (Figura 3), tem-se que esta rotina verifica se as entradas da alavanca analógica estão indicando a inclinação para alguma direção, gerando como saída a respectiva tecla do teclado. Em seguida o loop verifica se o push-button está pressionado, o qual ativa um LED para dar confirmação visual e analisa se o valor captado pelo sensor eletromagnético se encontra no intervalo definido (que ocorre quando o ímã passa sobre ele), se sim, o botão envia como saída a tecla " $Z$ " do teclado, que tem o papel de tecla de ação no jogo.

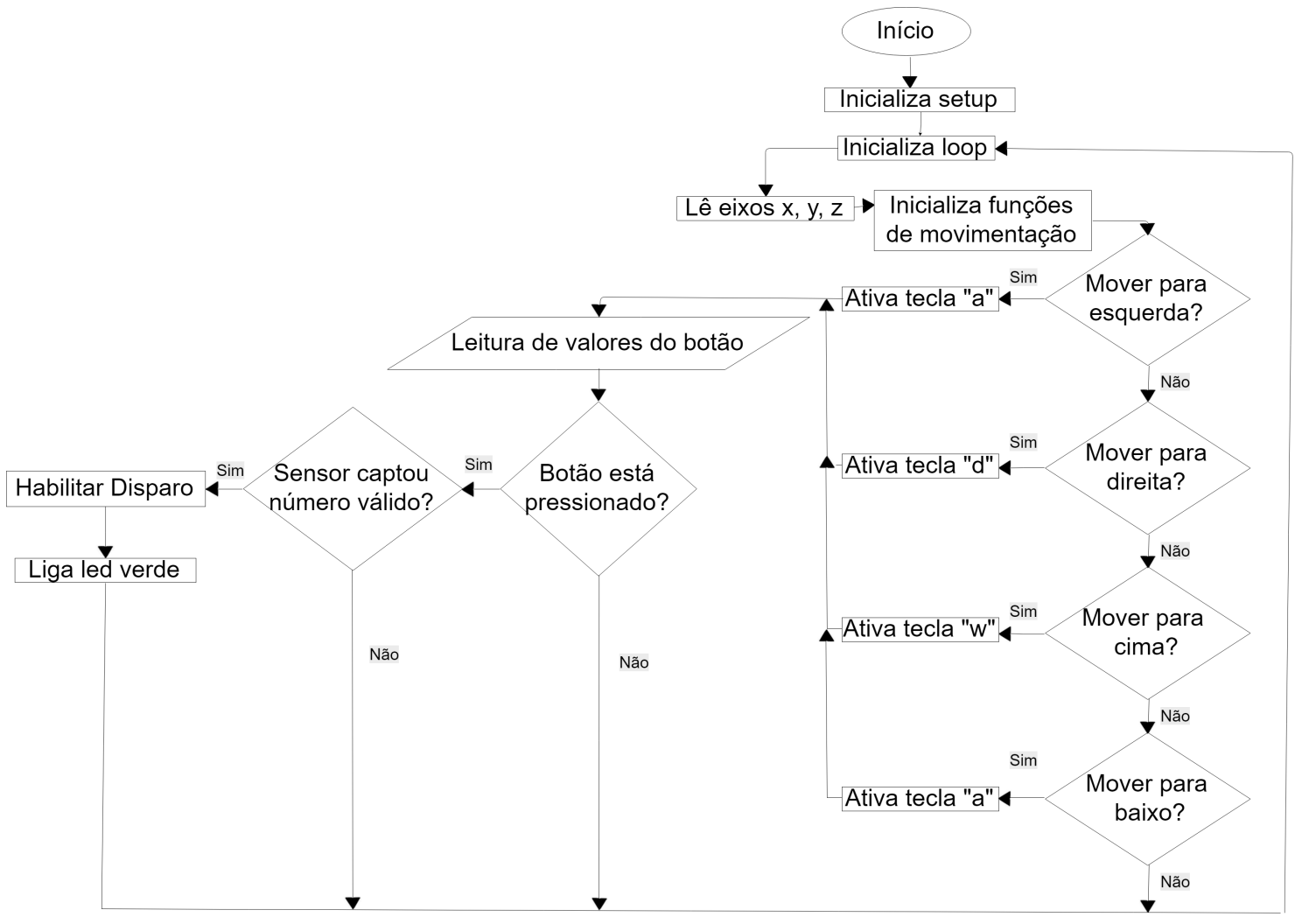

Figura 3. Esquema lógico de funcionamento do Cata-Sopro.

\section{Resultados \& Discussões}

Com relação aos custos envolvidos na confecção do protótipo inicial do Cata-Sopro, a Tabela 1 apresenta os valores atuais aproximados de cada um dos componentes aplicados. Tratam-se de componentes simples, baratos e de fácil acesso tanto em vendedores locais como em lojas virtuais de componentes eletrônicos, o que permite a fácil replicação do joystick proposto. 
Tabela 1. Componentes \& Valores utilizados na confecção do Cata-Sopro.

\begin{tabular}{|l|l|}
\hline Componente & Valor $(\mathbf{R} \$)$ \\
\hline Arduino Micro & aprox. 7,00 \\
\hline Push Button & aprox. $20,00(100$ unid. $)$ \\
\hline Sensor Magnético & aprox. 15,00 \\
\hline Resistor 10k & aprox. 10,00 $(20$ unid. $)$ \\
\hline Imãs & aprox. $19,00(100$ unid. $)$ \\
\hline Ventoinha & aprox. 10,00 \\
\hline
\end{tabular}

Com relação aos testes de verificação do Cata-Sopro, estes foram realizados pelos desenvolvedores do projeto, executando as operações básicas dos mesmo em 2 jogos distintos. No primeiro, foram verificadas mecânicas de movimentação de montaria no jogo "Mount Blade" (Figura 4(c)). Ao conectar o controle ao computador com o jogo "Mount Blade" aberto, o direcional analógico tomou, durante a partida, o papel das teclas "w, a, s, d" do teclado, ou seja, movia o personagem para frente, para trás e para os lados. Ao se pressionar o botão de ação juntamente com o sopro da hélice do controle, realizava-se a ação do botão "z" do teclado, que era o salto do cavalo. Para o segundo jogo de avaliação aplicado, efetuou-se disparos de uma nave no jogo "Space Invaders", através do sopro contínuo do jogador em conjunto com o pressionar do botão de ação do joystick (Figuras 4(a) e 4(b)). O direcional analógico ficou responsável pelo papel de movimentação lateral da nave na tela do jogo.

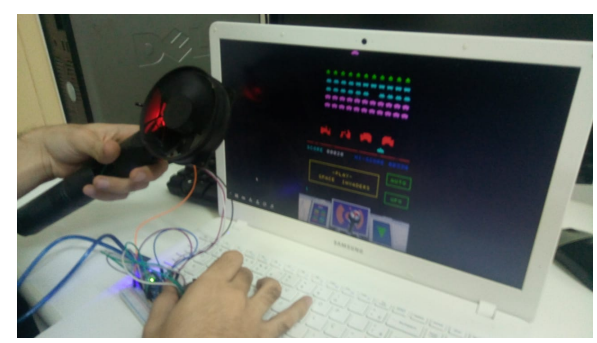

(a) Sensor de Tiro

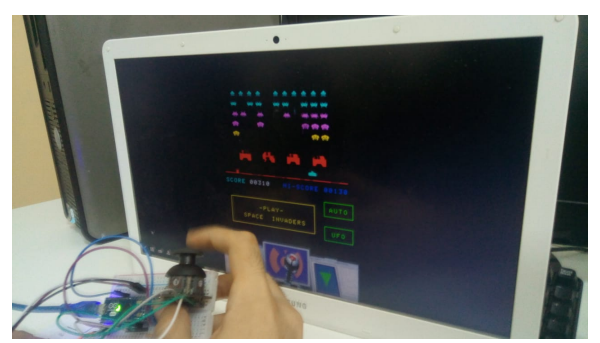

(b) Controle Direcional

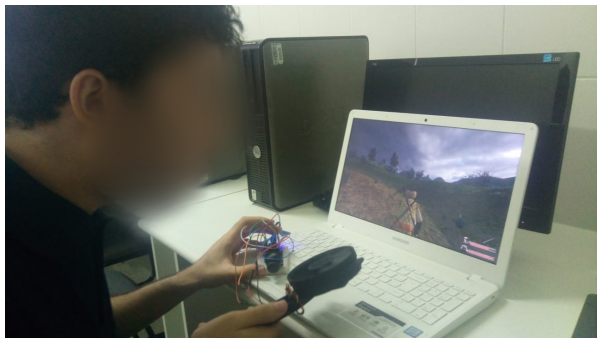

(c) Teste com o jogo Mount Blade

Figura 4. Testes de jogabilidade com o Cata-Sopro.

Em ambos os jogos de avaliação, os testadores encontraram dificuldades com o peso da hélice do controle, fazendo com que os mesmos precisassem de mais força no sopro para fazê-la girar. De fato, para se ter um bom uso do protótipo do joystick desenvolvido, é necessário ter potência de sopro o bastante para ser capaz de rodar o cooler que foi usado para sustentar os ímãs. 
O sopro contínuo para realizar vários disparos no Space Invaders também se mostrou inadequado e cansativo para os jogadores, mostrando a inviabilidade deste tipo de joystick em jogos que precisem efetuar muitas "disparos" ou "saltos" por exemplo. Como esse exercício contínuo pode levar a um rápido desgaste respiratório do jogador, é necessário rever o material das hélices de modo a substituí-lo por um mais leve e de fácil acesso, tal como um papel cartão ou um plástico mais fino.

\section{Conclusões e Trabalhos Futuros}

Este artigo apresentou o Cata-Sopro, um joystick de controle para jogos eletrônicos com funcionamento baseado em sopro e variação de campo magnético. Para tal, foram descritos o esquema de hardware, a lógica de funcionamento e os testes inicias de usabilidade aplicados no mesmo.

O joystick prototipado apresenta uma configuração simples e de baixo custo, facilitando assim a sua montagem e replicação por diferentes desenvolvedores. Ele também se apresenta como uma abordagem útil para a realização de exercícios repetitivos de sopro de uma maneira lúdica, algo de grande utilidade para a aplicação de exercícios de fortalecimento orofaciais realizados em terapias fonoaudiológicas com crianças e adolescentes.

Com trabalhos futuros, pretende-se efetuar confecções de jogos digitais com dinâmicas específicas para um melhor aproveitamento de diferentes rotinas de sopro. A confecção de uma case otimizada para o joystick proposto, bem como a realização de testes de usabilidade com mais jogadores de diferentes perfis e idades também serão realizados em um futuro próximo.

\section{Referências}

Alves, G. B., Sarinho, V. T., and Dias, A. M. (2018). Umistick: Developing a blow-based joystick using arduino sensors and a printed circuit board. XVII Simpósio Brasileiro de Jogos e Entretenimento Digital (SBGames).

Arroyo-Palacios, J. and Romano, D. M. (2009). Exploring the use of a respiratorycomputer interface for game interaction. In Games Innovations Conference, 2009. ICE-GIC 2009. International IEEE Consumer Electronics Society's, pages 154-159. IEEE.

Chen, W.-H. (2015). Blowatch: Blowable and hands-free interaction for smartwatches. In Proceedings of the 33rd Annual ACM Conference Extended Abstracts on human factors in computing systems, pages 103-108. ACM.

Clua, E. W. G. (2014). Jogos sérios aplicados a saúde. Journal of Health Informatics, 1.

Comin, I. and Passos Filho, L. P. d. P. (1999). Ortopedia funcional dos maxilares e fonoaudiologia: uma possibilidade terepêutica multidisciplinar. Rev. dent. press ortodon. ortop. maxilar, 4(4):63-70.

Patel, S. N. and Abowd, G. D. (2007). Blui: low-cost localized blowable user interfaces. In Proceedings of the 20th annual ACM symposium on User interface software and technology, pages 217-220. ACM.

Sarinho, V. T. (2017). Cornestick: Um joystick de sopro para jogos digitais. XVI Simpósio Brasileiro de Jogos e Entretenimento Digital (SBGames). 